\section{A mechanism of ubiquitin-independent proteasomal degradation of the tumor suppressors p53 and p73}

\author{
Gad Asher, Peter Tsvetkov, Chaim Kahana, and \\ Yosef Shaul $^{1}$ \\ Department of Molecular Genetics, Weizmann Institute of \\ Science, Rehovot 76100, Israel
}

Protein degradation is an essential and highly regulated process. The proteasomal degradation of the tumor suppressors $\mathrm{p} 53$ and $\mathrm{p} 73$ is regulated by both polyubiquitination and by an ubiquitin-independent process. Here, we show that this ubiquitin-independent process is mediated by the $20 \mathrm{~S}$ proteasomes and is regulated by NQO1. NQO1 physically interacts with p53 and p73 in an NADH-dependent manner and protects them from $20 S$ proteasomal degradation. Remarkably, the vast majority of NQO1 in cells is found in physical association with the $20 \mathrm{~S}$ proteasomes, suggesting that NQO1 functions as a gatekeeper of the $20 \mathrm{~S}$ proteasomes. We further show that this pathway plays a role in p53 accumulation in response to ionizing radiation. Our findings provide the first evidence for in vivo degradation of p53 and p73 by the $20 \mathrm{~S}$ proteasomes and its regulation by NQO1 and NADH level.

Supplemental material is available at http://www.genesdev.org.

Received August 4, 2004; revised version accepted December 7, 2004.

Protein degradation determines the outcome of many cellular physiological processes (Coux et al. 1996). Degradation of proteins by the proteasomes occurs via various pathways (Verma and Deshaies 2000; Pickart and Cohen 2004). The most intensely studied one is the ubiquitin-26S proteasome pathway (Hershko 1996; Hershko and Ciechanover 1998; Goldberg 2003). The tumor suppressor p53 is a very labile protein that undergoes $\mathrm{Mdm} 2$ and ubiquitin-dependent $26 \mathrm{~S}$ proteasomal degradation (Haupt et al. 1997; Kubbutat et al. 1997). Recently, we reported that degradation of p53 also occurs in an Mdm2 and ubiquitin-independent manner (Asher et al. 2002b). This pathway of p53 degradation is regulated by $\mathrm{NAD}(\mathrm{P}) \mathrm{H}$ quinone oxidoreductase 1 (NQO1) (Asher et al. 2001, 2002a,b, 2003, 2004), yet the underlying molecular mechanisms that control p53 degradation remained elusive.

\section{Results and Discussion}

To investigate the role of NQO1 in proteasomal degradation, we followed NQO1 distribution in fractionated mouse liver extracts. Ammonium sulfate precipitation and gel-filtration chromatography of liver extracts re-

[Keywords: NQO1; p73; p53; 20 S proteasome; protein degradation; NADH] ${ }^{1}$ Corresponding author.

E-MAIL yosef.shaul@weizmann.ac.il; FAX 972-8-9344108.

Article and publication are at http://www.genesdev.org/cgi/doi/10.1101/ gad.319905. vealed that the majority of NQO1 cofractionated with the $20 \mathrm{~S}$ proteasomes (Fig. 1A). These fractions are devoid of the $26 \mathrm{~S}$ proteasomes that were excluded by the differential ammonium sulfate precipitation (Fig. 1A, IB: 26S, anti TBP1 a subunit of the 19S). These results suggest that the vast majority of cellular NQO1 is found in a large protein complex that possibly includes the 20S proteasomes.

To further study this possibility, the 20S-containing fractions were pooled and fractionated by anion exchange chromatography according to a standard 20S purification protocol (Friguet et al. 2002). Remarkably, NQO1 was detected in the $0.3 \mathrm{M} \mathrm{NaCl}$ fraction containing the $20 \mathrm{~S}$ proteasomes (Fig. 1B). Electrophoresis of the $0.3 \mathrm{M} \mathrm{NaCl}$ fraction on a nondenaturing PAGE, followed by peptidase activity assay, showed that the purified $20 \mathrm{~S}$ is functional (Fig. 1C, Activity panel). Immunoblot analysis with anti NQO1 antibody revealed that NQO1 comigrated with the $20 \mathrm{~S}$ proteasomes, but not with the $26 \mathrm{~S}$ proteasomes (Fig. 1C). Finally, a coimmunopercipitation experiment showed that NQO1 coimmunopreciptated with $20 \mathrm{~S}$ proteasomes (Fig. 1D). Altogether, these results suggest that NQO1 and 20S proteasomes are in physical association. Immunoprecipitation of the $20 \mathrm{~S}$ proteasomes depleted most of the NQO1 from the supernatant (Fig. 1D), further indicating that the vast majority of cellular NQO1 is bound to the 20S proteasomes. Consistently, reticulocyte lysate translated ${ }^{35} \mathrm{~S}$-labeled NQO1-bound purified 20S proteasomes in vitro (Fig. 1E).

Analysis of p53 distribution in the gel-filtration fractions showed that a portion of p53 cofractionated with $20 \mathrm{~S}$ and NQO1 (Fig. 1A). Furthermore, both NQO1 and p53 were brought down following immunoprecipitation of the $20 \mathrm{~S}$ proteasomes, indicating the existence of a triplet p53-NQO1-20S complex (Fig. 1F).

The observed association of NQO1 with 20S proteasomes and our previous findings that NQO1 regulates p53 and p73 degradation prompted us to investigate the role of $20 \mathrm{~S}$ proteasomes in degradation of these proteins in vitro. To this end, reticulocyte lysate-translated ${ }^{35} \mathrm{~S}$ labeled p53 was incubated with purified 20S proteasomes and the level of ${ }^{35}$ S-labeled p53 was determined by SDS-PAGE and autoradiography. A substantial amount of p53 was degraded already after $15 \mathrm{~min}$, and this was inhibited in the presence of the proteasome inhibitor MG132 (Fig. 2A). Similar results were obtained with ${ }^{35}$ S-labeled p73 $\alpha$ (Fig. 2B). p53 and p73 degradation was not observed in the presence of purified $26 \mathrm{~S}$ proteasomes (data not shown). To rule out the involvement of reticulocyte lysate components in degradation by the $20 \mathrm{~S}$ proteasomes, the ${ }^{35}$ S-labeled p73 was first immunoaffinity purified (Supplementary Fig. 1) and then subjected to degradation by the $20 \mathrm{~S}$ proteasomes. Under these conditions, a similar degree of p73 degradation was observed (Fig. 2C).

Next, we addressed the involvement of NQO1 in $20 \mathrm{~S}$ proteasomal degradation. The in vitro degradation assays were repeated in the absence or presence of in vitrotranslated NQO1. Remarkably, p53 and p73 $\alpha$ degradation by the $20 \mathrm{~S}$ proteasomes was inhibited in the presence of excess NQO1 and NADH, a cofactor of NQO1 (see below), suggesting that NQO1 directly regulates the degradation of these proteins by the $20 \mathrm{~S}$ proteasomes (Fig. 2D,E). In contrast, $20 \mathrm{~S}$ degradation of $\mathrm{p} 73 \beta$, a p73 isoform that lacks the C-terminal SAM do- 
A
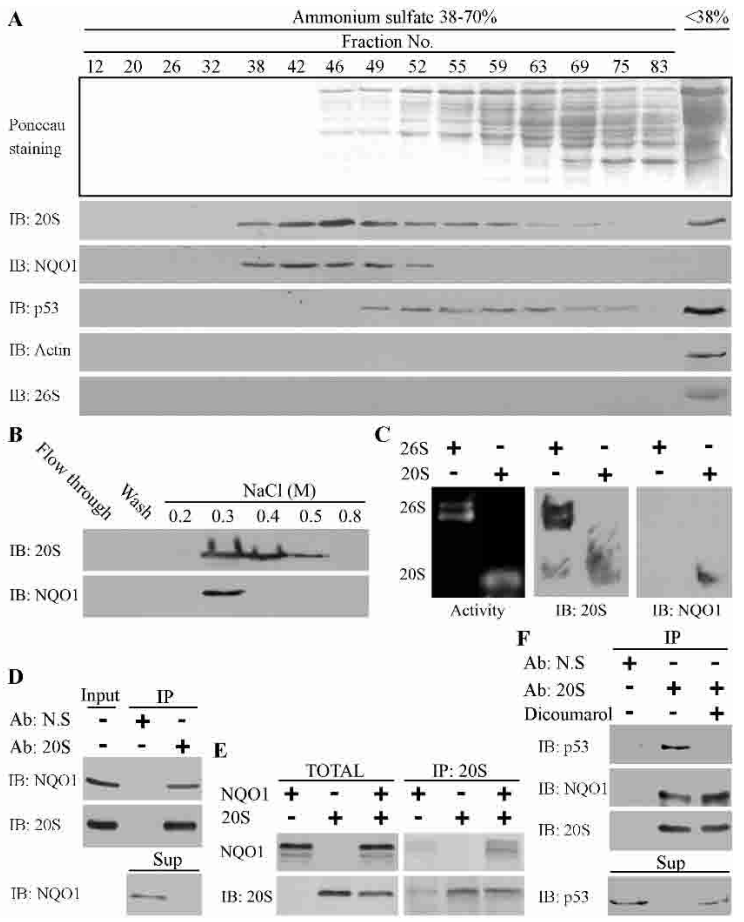

Figure 1. NQO1 is physically associated with $20 \mathrm{~S}$ but not $26 \mathrm{~S}$ proteasomes. (A) Mouse liver extract was precipitated at 38\%-70\% ammonium sulfate and subjected to Sepharose 6B gel-filtration chromatography. Fractions were collected and analyzed by SDSPAGE and immunoblotting (IB). (B) Fractions from Sepharose 6B gel-filtration column containing 20S proteasomes were loaded on Resource Q anion-exchange column and eluted with different concentrations of $\mathrm{NaCl}$. Fractions were analyzed by SDS-PAGE and immunoblotting (IB). (C) Purified 26S and 20S proteasomes $(0.3 \mathrm{M}$ $\mathrm{NaCl}$ fraction) were subjected to nondenaturing PAGE and analyzed for peptidase activity or by immunoblotting (IB). (D) Fractions from Sepharose 6B gel-filtration column containing 20S proteasomes were pooled (Input), and immunoprecipitation experiments were performed with mouse anti-Flag antibody as a control for nonspecific binding (Ab: N.S) or with rabbit anti-C9 antibody (Ab: 20S). The immunoprecipitants (IP) and the supernatant (Sup) were analyzed by SDS-PAGE and immunoblotting (IB) $(E)^{35}$ S-labeled NQO1 was incubated alone or mixed together with $20 \mathrm{~S}$ proteasomes (TOTAL). The $20 \mathrm{~S}$ proteasomes were immunoprecipitated with rabbit anti-C 9 antibody (IP: 20S). (F) Fractions from Sepharose 6B gel-filtration column containing $20 \mathrm{~S}$ proteasomes were pooled and immunoprecipitation experiments were performed with mouse anti-Flag antibody as a control for nonspecific binding (Ab: N.S) or with rabbit anti-C9 antibody (Ab: 20S) in the absence $(-)$ or presence $(+)$ of $200 \mu \mathrm{M}$ dicoumarol. The immunoprecipitants (IP) and the supernatant (Sup) were analyzed by SDS-PAGE and immunoblotting (IB). Immunoblot analysis (IB) was performed with rabbit anti-C9 antibody to identify the $20 \mathrm{~S}$ proteasomes, rabbit anti-TBP1 antibody to identify the $26 \mathrm{~S}$ proteasomes, goat anti-NQO1 antibody, mouse anti-mouse p53 antibody, and with mouse anti-Actin. ${ }^{35}$ S-labeled NQO1 was detected by autoradiography. Proteasome peptidase activity was determined by their ability to hydrolyze the flurogenic peptide suc-LLVY-AMC, as described in Materials and Methods.

main region, was not affected by NQO1 (Fig. 2E). This distinctive behavior was also observed in vivo in cells overexpressing NQO1 by accumulation of endogenous $\mathrm{p} 73 \alpha$ and $\mathrm{p} 53$, but not of $\mathrm{p} 73 \beta$ (Fig. 2F). These observations are in agreement with the finding that NQO1 knockout mice display reduced levels of $\mathrm{p} 73 \alpha$ and $\mathrm{p} 53$ (Long et al. 2002).

The observation that $\mathrm{p} 73 \beta$ degradation is NQO1 re- fractory led us to investigate the requirement of the Cterminal SAM domain region in $\mathrm{p} 73 \alpha$ stabilization by NQO1. We and others have previously reported that NQO1 binds p53 (Anwar et al. 2003; Asher et al. 2003). To examine whether NQO1 binds p73 $\alpha$ through its unique C-terminal region, Flag-p $73 \alpha$, Flag-p $73 \beta$, and Flag-p73 $\Delta 1-317$ were expressed either alone or together with NQO1 in 293 human kidney (HEK) cells; cell extracts were subjected to immunoprecipitation with an anti-Flag antibody. NQO1 coimmunoprecipitated with wild-type $\mathrm{p} 73 \alpha$ and with the $\mathrm{N}$-terminal truncated

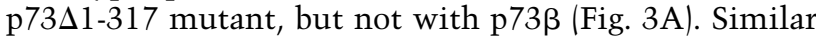
results were obtained using in vitro binding assays with

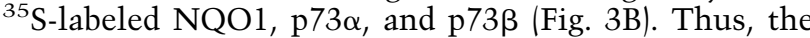
$\mathrm{C}$-terminal SAM domain region of $\mathrm{p} 73 \alpha$ is required for direct binding to NQO1 both in vivo and in vitro. These results suggest that the interaction between NQO1 and $\mathrm{p} 73 \alpha$ protect $\mathrm{p} 73 \alpha$ from degradation by the $20 \mathrm{~S}$ proteasomes.

Next, we performed in vitro binding assays in the presence of the NQO1 cofactors NAD, FAD, or NADH. A remarkable increase in the binding of NQO1 to $\mathrm{p} 73 \alpha$ was observed only in the presence of NADH (Fig. 3C). Similarly, the binding of NQO1 to p53 was increased in the

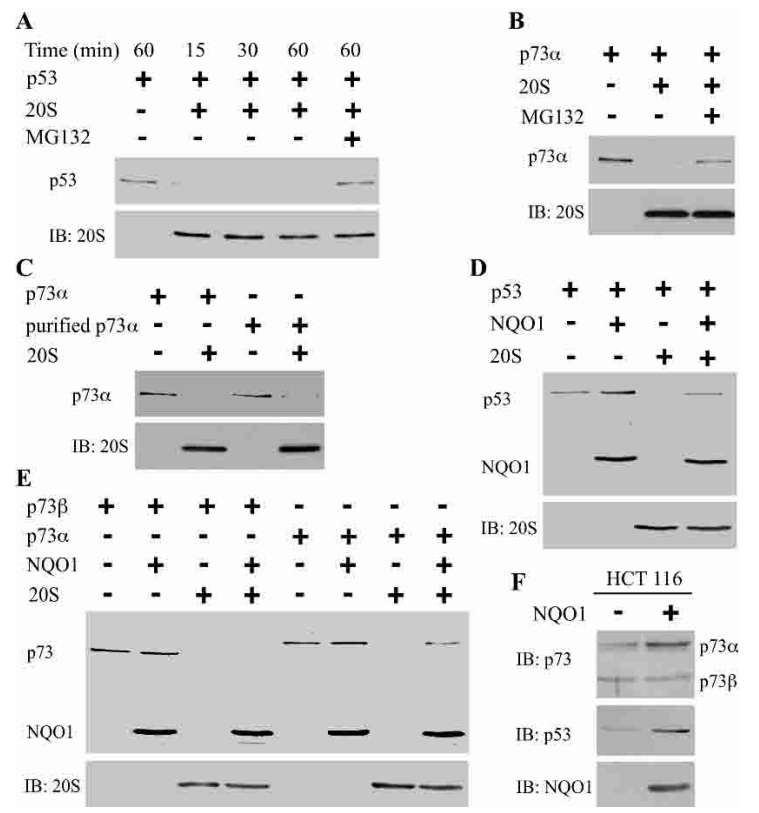

Figure 2. NQO1 selectively protects $\mathrm{p} 73 \alpha$, but not $\mathrm{p} 73 \beta$, from $20 \mathrm{~S}$ proteasomal degradation. $(A)^{35}$ S-labeled p53 was incubated without $(-)$ or with $(+) 20 \mathrm{~S}$ proteasomes at $37^{\circ} \mathrm{C}$ for 15,30 , and $60 \mathrm{~min}$, without $(-)$ or with $(+) 50 \mu \mathrm{M}$ MG132. $(B){ }^{35}$ S-labeled p73 $\alpha$ was incubated without $(-)$ or with $(+) 20$ S proteasomes without $(-)$ or with $(+) 50 \mu \mathrm{M}$ MG132 at $37^{\circ} \mathrm{C}$ for $1 \mathrm{~h} .(\mathrm{C}){ }^{35}$ S-labeled Flag-p73 $\alpha$ or immunoaffinity-purified ${ }^{35}$ S-labeled Flag-p $73 \alpha$ were incubated without $(-)$ or with $(+) 20 \mathrm{~S}$ proteasomes at $37^{\circ} \mathrm{C}$ for $1 \mathrm{~h} .(D)^{35} \mathrm{~S}$-labeled p53 was incubated in the presence of $1 \mathrm{mM}$ NADH without $(-)$ or with $(+)$ 20S proteasomes, without $(-)$ or with $(+){ }^{35}$ S-labeled NQO1 $(E)^{35}$ S-labeled $\mathrm{p} 73 \alpha$ or $\mathrm{p} 73 \beta$ were incubated in the presence of $1 \mathrm{mM}$ NADH without $(-)$ or with $(+) 20$ S proteasomes, without (-) or with $(+){ }^{35}$ S-labeled NQO1. ${ }^{35}$ S-labeled p73 and NQO1 were analyzed by SDS-PAGE and detected by autoradiography. The $20 \mathrm{~S}$ proteasomes were detected by immunoblot analysis (IB) with rabbit anti C9 antibody. $(F)$ Cell extracts of HCT116 cells and HCT116 cells that stably express pEFIRES NQO1 were fractionated by SDS-PAGE; immunoblot analysis (IB) was carried out with mouse anti-p53, with rabbit anti-p73 antibody, and with goat anti-NQO1 antibody. 


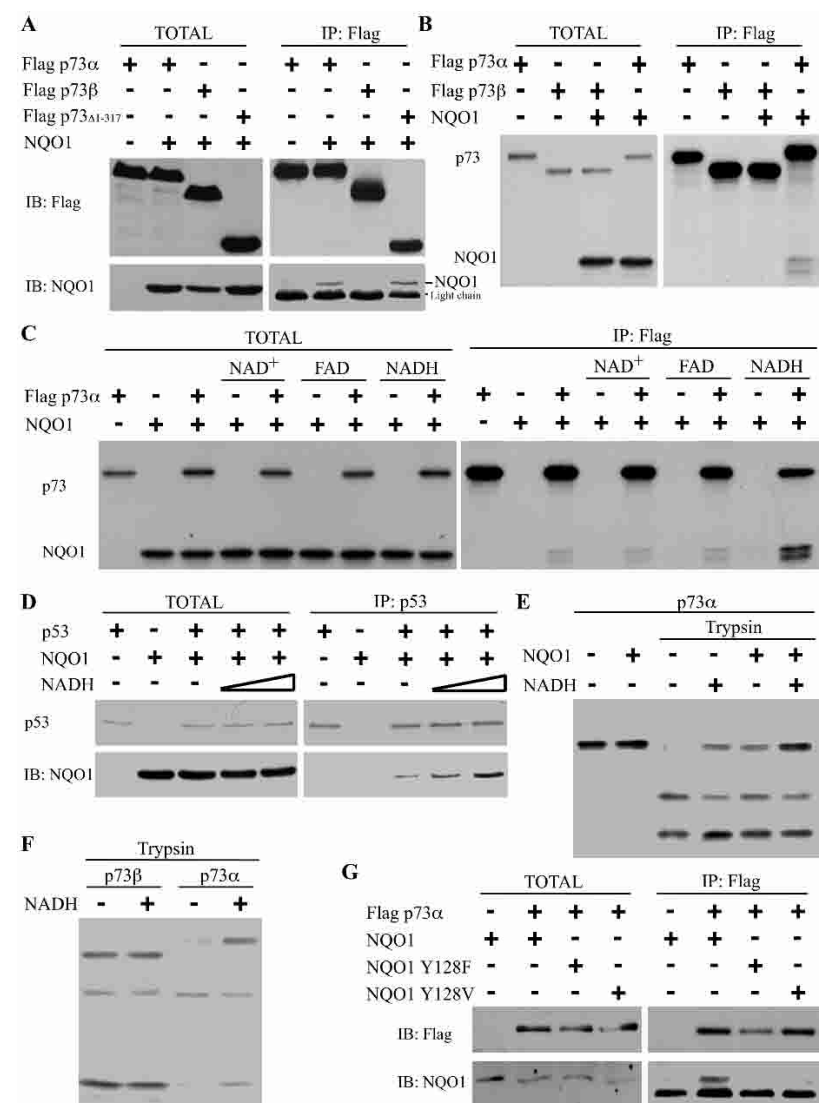

Figure 3. NQO1 binds the SAM domain of p73 $\alpha$ in an NADHdependent manner. (A) 293 HEK cells were transiently transfected with pEFIRES Flag-p73 $\alpha$, pEFIRES Flag-p73 $\beta$, or pEFIRES Flagp73 1-317 without (-) or with pEFIRES-NQO1 (TOTAL). Flag-p73 was immunoprecipitated with anti-Flag beads (IP: Flag). (B) In vitro ${ }^{35}$ S-labeled Flag-p $73 \alpha$ or Flag-p $73 \beta$ were incubated alone or mixed together with ${ }^{35}$ S-labeled NQO1 (TOTAL). Flag-p73 was immunoprecipitated with anti-Flag beads (IP: Flag). $(C)^{35}$ S-labeled Flag-p $73 \alpha$ and NQO1 were incubated alone or mixed together without or with $\mathrm{NAD}^{+}, \mathrm{FAD}$, or NADH (TOTAL). Flag-p73 was immunoprecipitated with anti-Flag beads (IP: Flag). ( $D$ ) In vitro ${ }^{35}$ S-labeled p53 was incubated alone $(-)$ or together with $(+)$ recombinant NQO1 in the absence (-) or presence of $100 \mu \mathrm{M}$ or $1 \mathrm{mM}$ NADH. p53 was immunoprecipitated with mouse anti-human p53 antibody (IP: p53). (E) In vitro ${ }^{35}$ S-labeled Flag-p $73 \alpha$ was incubated without $(-)$ or with $(+) 1$ $\mathrm{mM} N \mathrm{NADH}$, in vitro-translated NQO1 or $1 \mathrm{mM} \mathrm{NADH}$ together with in vitro-translated NQO1, and partially digested with trypsin. (F) In vitro ${ }^{35}$ S-labeled Flag-p $73 \alpha$ or Flag-p $73 \beta$ was incubated without (-) or with (+) $1 \mathrm{mM}$ NADH and partially digested with trypsin. (G) 293 HEK cells were transiently transfected with pEFIRES Flagp73 $\alpha$ alone or together with pEFIRES NQO1, pEFIRES NQO1Y128F, or pEFIRES NQO1-Y128V (TOTAL). Flag-p73 was immunoprecipitated with anti-Flag beads (IP: Flag). Immunoblot analysis (IB) was carried out with mouse monoclonal anti-Flag antibody and with goat anti-NQO1 antibody. ${ }^{35}$ S-labeled NQO1, p53, and p73 were detected by autoradiography.

presence of $\mathrm{NADH}$ in a dose-dependent manner (Fig. 3D). To test the possible effect of NQO1 and NADH on p73 conformation, we performed partial trypsin digestion experiments. Partial trypsin digestion of ${ }^{35} \mathrm{~S}$-labeled p $73 \alpha$ was inhibited in the presence of NADH or NQO1, and to a greater extent, in the presence of both NADH and NQO1 (Fig. 3E). These results suggest that NQO1 introduces a conformational change in p73 $\alpha$ that is augmented in the presence of NADH. The effect of NADH alone may be due to NQO1 found in the lysate (data not shown), however, a direct effect of NADH on p73 $\alpha$ conformation cannot be ruled out. Digestion of $\mathrm{p} 73 \alpha$, but not $\mathrm{p} 73 \beta$, was inhibited in the presence of NADH, indicating that this effect is mediated via the p $73 \alpha \mathrm{C}$-terminal SAM domain region (Fig. 3F). To examine the requirement of NADH for NQO1 binding to $\mathrm{p} 73 \alpha$ in vivo, we utilized both genetic and pharmacological approaches. Tyr 128 of NQO1 is peripherally involved in the binding of NADH (Ma et al. 1992). Therefore, we examined the ability of $\mathrm{p} 73 \alpha$ to bind two different NQO1 mutants with reduced NADH-binding capacity, NQO1Y128F and NQO1-Y128V (Ma et al. 1992). In contrast to wild-type NQO1, neither NQO1-Y128F nor NQO1Y128V coimmunoprecipitated with $\mathrm{p} 73 \alpha$ in 293 HEK cells (Fig. 3G), confirming the requirement of NQO1associated $\mathrm{NADH}$ for binding to $\mathrm{p} 73 \alpha$ in vivo.

Dicoumarol, a drug that competes specifically with NADH for binding to NQO1 (Hosoda et al. 1974), was used to further confirm the requirement of $\mathrm{NADH}$ for the p73-NQO1 interaction. The in vitro binding assay with ${ }^{35}$ S-labeled NQO1 and p $73 \alpha$ was performed in the absence or presence of NADH, and without or with dicoumarol. The binding of $\mathrm{p} 73 \alpha$ to NQO1 was increased in the presence of NADH and decreased upon addition of dicoumarol (Fig. 4A). To recapitulate these experiments in cells, coimmunoprecipitated p $73 \alpha-$ NQO1 complex was washed with dicoumarol before its elution and separation on SDS-PAGE. The level of NQO1 associated with p $73 \alpha$ was strongly reduced by dicoumarol (Fig. 4B), suggesting that dicoumarol dissociates the preformed p73 $\alpha$-NQO1 complex. Similar results were obtained with p53 (see Fig. 5A).

Our findings suggest that the NADH-dependent binding of NQO1 to $\mathrm{p} 73 \alpha$ protect $\mathrm{p} 73 \alpha$ from 20 S proteasomal degradation. Since dicoumarol dissociates the NQO1$\mathrm{p} 73 \alpha$ complex, it is expected to promote p $73 \alpha$ degradation. Indeed, in vitro degradation of ${ }^{35}$ S-labeled $p 73 \alpha$ was enhanced in the presence of dicoumarol (Fig. 4C). Conversely, p73 $\alpha$ was stabilized in the presence of NQO1 (Fig. 4D), but not by NQO1-Y128F or NQO1-Y128V mutants that did not bind $\mathrm{p} 73 \alpha$ (Supplementary Fig. 2). Furthermore, in the presence of dicoumarol, NQO1 no longer protected $\mathrm{p} 73 \alpha$ from degradation by the $20 \mathrm{~S}$ proteasomes (Fig. 4E). The effect of dicoumarol was also tested in vivo. Treatment of either HCT116 human colon carcinoma cells or COS-1 cells with dicoumarol resulted in a dose-dependent decrease in the level of endogenous $\mathrm{p} 73 \alpha$, but not of $\mathrm{p} 73 \beta$, which does not bind NQO1 (Fig. 4F). Consistently, dicoumarol induced degradation of HA-p $73 \alpha$, but not of HA-p $73 \beta$ or HAp73 $493-521$, a deletion mutant that lacks the C-terminal SAM domain expressed in 293 HEK cells (Fig. 4G). The decrease in the level of $\mathrm{p} 73 \alpha$ following dicoumarol treatment was prevented by the presence of the proteasome inhibitor lactacystin (Fig. 4H). Finally, by using the A31N-ts20 BALB/c cell line that harbors a temperaturesensitive E1 variant and, therefore, defective in polyubiquitination at the restrictive temperature (Chowdary et al. 1994), we show that dicoumarol-induced $\mathrm{p} 73 \alpha$ and p53 degradation is ubiquitin independent (Fig. 4I; Asher et al. 2002b), supporting the possibility that this process is executed in vivo by the $20 \mathrm{~S}$ proteasomes.

Dicoumarol inhibits the accumulation of p53 following $\gamma$-irradiation (IR) and blocks p53-dependent apoptosis in mouse thymocites (Asher et al. 2001). To examine 


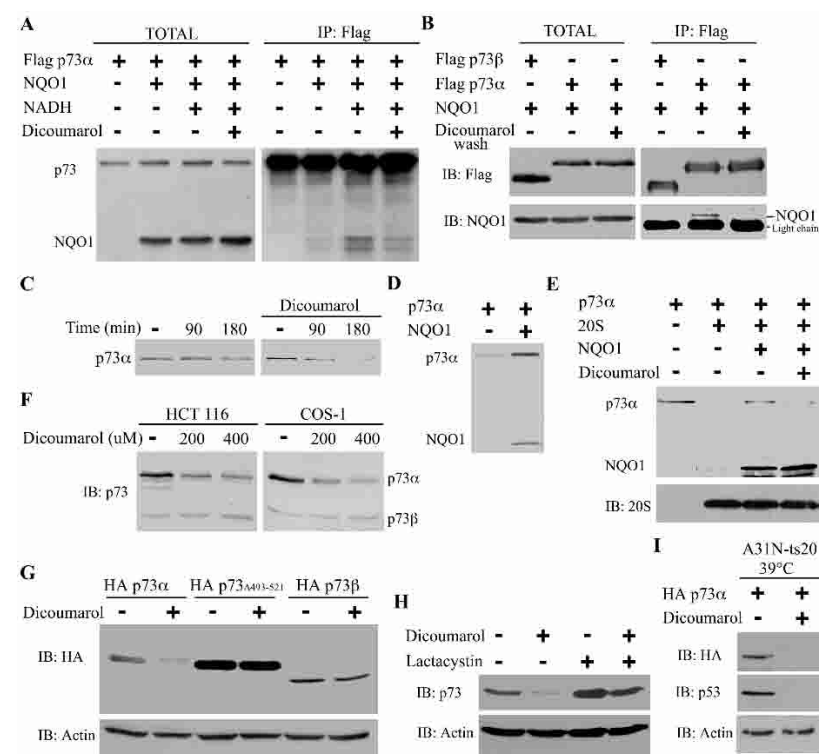

Figure 4. Dicoumarol disrupts the binding of NQO1 to $\mathrm{p} 73 \alpha$ and induces ubiquitin-independent proteasomal degradation of $\mathrm{p} 73 \alpha .(A)$ ${ }^{35}$ S-labeled Flag-p $73 \alpha$ was incubated alone or with NQO1 in the absence (-) or presence of (+) NADH or NADH together with $300 \mu \mathrm{M}$ dicoumarol (TOTAL). Flag-p73 was immunoprecipitated with antiFlag beads (IP: Flag). (B) 293 HEK cells were transiently transfected with pEFIRES Flag-p $73 \beta$ or pEFIRES Flag-p $73 \alpha$ together with pEFIRES NQO1 (TOTAL). Flag-p73 was immunoprecipitated with anti-Flag beads (IP: Flag). The beads were washed without (-) or with $(+) 300 \mu \mathrm{M}$ dicoumarol (dicoumarol wash). $(C)^{35}$ S-labeled p73 $\alpha$ was incubated in reticulocyte lysate degradation mixture at $37^{\circ} \mathrm{C}$ for 90 and $180 \mathrm{~min}$ in the absence or presence of $300 \mu \mathrm{M}$ dicoumarol. $(D){ }^{35}$ S-labeled Flag-p $73 \alpha$ was incubated alone or together with ${ }^{35} \mathrm{~S}$ labeled NQO1 in reticulocyte lysate degradation mixture at $37^{\circ} \mathrm{C}$ for $90 \mathrm{~min} .(E)^{35} \mathrm{~S}$-labeled $\mathrm{p} 73 \alpha$ was incubated in the presence of $\mathrm{NADH}$ without $(-)$ or with $(+) 20$ S proteasomes without $(-)$ or with $(+)$ ${ }^{35}$ S-labeled NQO1 and without $(-)$ or with $(+) 200 \mu \mathrm{M}$ dicoumarol. (F) HCT116 or COS 1 cells were cultured without (-) or with 200 or $400 \mu \mathrm{M}$ dicoumarol for $5 \mathrm{~h} .(G)$ HCT1 16 cells were transiently transfected with pSG5 HA-p73 $\alpha$, pSG5 HA-p73 4493-521, or pSG5 HA-p $73 \beta$, and 24 -h post transfection, cells were cultured without (-) or with $(+) 300 \mu \mathrm{M}$ dicoumarol for $5 \mathrm{~h}$. $(H)$ HCT116 cells were cultured without $(-)$ or with $(+) 200 \mu \mathrm{M}$ dicoumarol and without $(-)$ or with $(+) 50 \mu \mathrm{M}$ lactacystin for $5 \mathrm{~h}$. (I) A31N-ts20 cells were transiently transfected with pSG5 HA-p $73 \alpha$, incubated for $24 \mathrm{~h}$ at the restrictive temperature $\left(39^{\circ} \mathrm{C}\right)$, and then cultured for $5 \mathrm{~h}$ without $(-)$ or with $(+) 300 \mu \mathrm{M}$ dicoumarol. Immunoblot analysis (IB) was carried out with the following antibodies: mouse anti-Flag, mouse antiHA, goat anti-NQO1, rabbit anti-p73, mouse anti-mouse p53, mouse anti-Actin, and rabbit anti-C9 to identify the $20 \mathrm{~S}$ proteasomes. ${ }^{35}$ S-labeled p73 and NQO1 were detected by autoradiography.

whether the binding of p53 and p $73 \alpha$ to NQO1 is increased following IR, Flag-p53 or Flag-p73 $\alpha$ and NQO1 were expressed in 293 HEK cells and cells were exposed to IR. Coimmunoprecipitation experiments revealed that under these conditions, the binding of $\mathrm{p} 53$ and $\mathrm{p} 73 \alpha$ to NQO1 is increased (Fig. 5A,B). The increase in NQO1-p53 binding is NADH dependent, as the complex dissociated in the presence of dicoumarol (Fig. 5A). Dicoumarol treatment also reduced $\mathrm{p} 53$ and $\mathrm{p} 73 \alpha$ accumulation following IR (Fig. 5C), suggesting that NQO1 binding is important for p53 and p73 $\alpha$ stabilization under these conditions. The role of Mdm2 in ubiquitin and 26S proteasomal p53 degradation is well documented. Upon $\gamma$-irradiation, p53 undergoes post-translational modifications and escapes Mdm2-mediated degradation (Vogel- stein et al. 2000). Interestingly, when A31N-ts20 cells were $\gamma$-irradiated under conditions defective in polyubiquitination, whereby $\mathrm{Mdm} 2$ can no longer promote p53 degradation (Asher et al. 2002b), the level of p53 following IR was further increased (Fig. 5D), suggesting that p53 accumulates independently of ubiquitin-mediated degradation. Furthermore, similar results were obtained with human $\mathrm{p} 53^{[22,23]}$, a p53 mutant that does not bind Mdm2 (Fig. 5E). Coexpression of NQO1-specific siRNA (Asher et al. 2002b) with human p53 ${ }^{[22,23]}$ prevented p53 accumulation following IR (Fig. 5E). Our results indicate that NQO1 plays a role in p53 and p $73 \alpha$ accumulation following $\gamma$-irradiation. Escaping Mdm2-mediated degradation is probably not sufficient for efficient p53 stabilization following IR, because p53 is still susceptible to $20 \mathrm{~S}$ proteasomal degradation. We propose that in order to
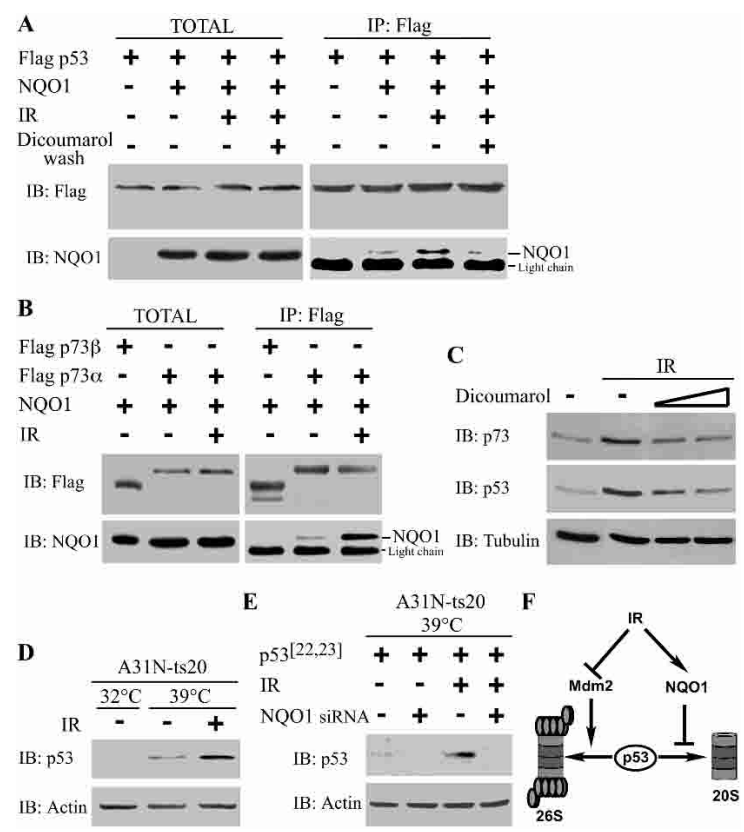

Figure 5. NQO1 stabilizes p $73 \alpha$ and p53 following $\gamma$-irradiation. (A) 293 HEK cells were transiently transfected with pRc/CMV Flagp53 without (-) or with (+) pEFIRES NQO1. Twenty-four hours following transfection, cells were $\gamma$-irradiated at 4 Gy (IR) and cultured for an additional $4 \mathrm{~h}$ (TOTAL). Flag-p53 was immunoprecipitated with anti-Flag beads (IP: Flag). The beads were washed without (-) or with $(+) 300 \mu \mathrm{M}$ dicoumarol (dicoumarol wash). (B) 293 HEK cells were transiently transfected with pEFIRES Flag-p $73 \beta$ or pEFIRES Flag-p73 $\alpha$, together with pEFIRES NQO1. Twenty-four hours following transfection, cells were $\gamma$-irradiated at 4 Gy (IR) and cultured for an additional $4 \mathrm{~h}$ (TOTAL). Flag-p73 was immunoprecipitated with anti-Flag beads (IP: Flag). (C) HCT116 cells were $\gamma$-irradiated at $6 \mathrm{~Gy}$ (IR) and cultured without (-) or with 200 or $400 \mu \mathrm{M}$ dicoumarol for $4 \mathrm{~h}$. (D) A31N-ts20 cells were incubated for $24 \mathrm{~h}$ at the permissive $\left(32^{\circ} \mathrm{C}\right)$ or restrictive $\left(39^{\circ} \mathrm{C}\right)$ temperature. Cells cultured at $39^{\circ} \mathrm{C}$ were then $\gamma$-irradiated at $4 \mathrm{~Gy}$ (IR) and cultured for additional $4 \mathrm{~h}$ at $39^{\circ} \mathrm{C}$. (E) A31N-ts 20 cells were transiently transfected with $\mathrm{pRc} /$ CMV human p53 $3^{[22,23]}$ without or with pSUPER NQO1 encoding NQO1-specific siRNA. Cells were then incubated for $24 \mathrm{~h}$ at $39^{\circ} \mathrm{C}$, $\gamma$-irradiated at $4 \mathrm{~Gy}(\mathrm{IR})$, and cultured for an additional $4 \mathrm{~h}$ at $39^{\circ} \mathrm{C}$. (F) A schematic model of p53 accumulation following IR. Protein extraction and immunoblot analysis (IB) were carried out as described in the Materials and Methods with mouse anti-mouse p53, mouse anti-human p53, mouse anti-Flag antibody, goat anti-NQO1 antibody, rabbit anti-p73, and mouse anti-Actin or anti-Tubulin antibodies. 
achieve efficient p53 accumulation following $\gamma$-irradiation, NQO1-p53 interaction is increased to eliminate p53 degradation by the $20 \mathrm{~S}$ proteasomes (Fig. 5F).

This study provides evidence for in vivo $20 \mathrm{~S}$ proteasomal degradation of specific short-lived proteins. First, unlike the $26 \mathrm{~S}$ proteasomal degradation, the pathway described here does not require polyubiquitination. Second, this pathway is responsive in vivo to inhibitors of the core $20 \mathrm{~S}$ proteasomes, such as MG132 and lactacystin, but is not inhibited by glucoseamine /data not shown), an inhibitor of the $26 \mathrm{~S}$ proteasomes (Zhang et al. 2003). Third, the observed in vivo process was fully and reliably recapitulated in vitro using purified $20 \mathrm{~S}$ proteasomes. Under both conditions, $\mathrm{p} 73 \alpha$ and p53 degradation was blocked by NQO1 in the presence of NADH and stimulated by dicoumarol. Recent experiments in our lab suggest that NQO1 also binds and stabilizes ornithine decarboxilase (ODC) in vivo (data not shown). Remarkably, all of these NQO1-binding proteins undergo $20 \mathrm{~S}$ proteasomal degradation in vitro and are protected by NQO1. Unlike p53, p73 $\alpha$, and ODC, the stability of other short-lived proteins, such as the cyclin D1 and p27 ${ }^{\text {kip }}$, was not affected by NQO1 (Supplementary Fig. 3).

Our findings suggest that NQO1 functions as a gatekeeper of the $20 \mathrm{~S}$ proteasomes. NQO1 is associated with the $20 \mathrm{~S}$ proteasomes and interacts with p $73 \alpha$ and p53 in an NADH-dependent manner to protect them from $20 \mathrm{~S}$ proteasomal degradation. Dicoumarol, by competing with NADH, abolishes the NQO1-p73 $\alpha$ and p53 interaction and, therefore, sensitizes these proteins to degradation by the $20 \mathrm{~S}$ proteasomes. Dicoumarol does not alter the binding of NQO1 to the 20S proteasomes (Supplementary Fig. 4). Indeed, treatment of the 20S-NQO1-p53 ternary complexes with dicoumarol resulted in dissociation only of p53, but not of NQO1 and 20S (Fig. 1F). The interaction of NQO1 with p53 and p73, potential $20 \mathrm{~S}$ substrates, is modulated under various physiological conditions, such as IR (Fig. 5F).

\section{Materials and methods}

\section{Cells and cell culture}

The cell lines used were as follows: 293 human kidney cells (HEK), COS-1 monkey kidney, HCT116 human colon carcinoma, p53 null HCT116 cells, HCT116 stably transfected with NQO1 (Asher et al. 2001), M1-t-p53 mouse myeloid leukemic cells (Asher et al. 2001), and A31N-ts20, a BALB/c mouse cell line that harbors a temperature-sensitive E1 ubiquitin-activating enzyme (Chowdary et al. 1994). Cells were grown as previously described (Asher et al. 2002b).

\section{Compounds}

Dicoumarol (Sigma) was dissolved in $0.13 \mathrm{~N} \mathrm{NaOH}$, lactacystin (Sigma) in DMSO. $\beta$-NADH, $\beta-\mathrm{NAD}^{+}$, and FAD (Sigma) were dissolved in water.

\section{Plasmids and transfection}

The plasmids used were as follows: pEFIRES and pSG5 NQO1 encoding wild-type human NQO1, pEFIRES and pSG5 NQO1-Y128F, pEFIRES and pSG5 NQO1-Y128V, pSUPER NQO1 encoding NQO1-specific siRNA, pSG5 HA-p $73 \alpha$ encoding wild-type monkey p73, pSG5 HA-p73 $\beta$, pSG5 HA-p 73 $\Delta 493-521$, pEFIRES Flag-p $73 \alpha$, pEFIRES Flag-p $73 \beta$, pEFIRES Flagp73 1-317, pRc/CMV p53 encoding wild-type human p53, and pRc/CMV p53 $3^{[22,23]}$. Transient transfections of 293 human kidney cells were carried out by the calcium phosphate method. Transfection of A31N-ts20 cells was carried out with jetPEI, (Poly Transfection).

Immunoblot analysis

Cell extracts and immunoblot analysis were carried out as previously described (Asher et al. 2001). The antibodies used were as follows: goat
anti-NQO1 C19 and R20 (Santa Cruz), mouse anti-human p53 (Pab 1801), mouse anti-mouse and human p53 (Pab 240), mouse anti-cyclin D1 (Santa Cruz), rabbit anti-p27 $7^{\text {kip }}$ antibody (Santa Cruz), rabbit anti-p73 (Asher et al. 2002b), rabbit anti-TBP1 (a subunit of the 19S), rabbit antiC9 subunit of the 20S proteasomes (Mamroud-Kidron et al. 1994), mouse monoclonal anti-HA (Sigma), mouse monoclonal anti-Flag (Sigma), and mouse monoclonal anti-Actin (Sigma).

\section{In vitro protein degradation assays}

The in vitro degradation assay of in vitro reticulocyte lysate-translated $\left[{ }^{35} \mathrm{~S}\right]$ methionine $\mathrm{p} 73 \alpha$ was performed as previously described (Asher et al. $2002 \mathrm{~b})$. Degradation of in vitro-translated $\left[{ }^{35} \mathrm{~S}\right]$ methionine p73 or p53 with $1 \mu \mathrm{g}$ of purified 20S proteasome (from Sigma, or prepared by us, or kindly provided by Dr. Y. Reiss, Proteologies, Ltd., Rehovot, Israel) was carried out in degradation buffer (100 mM Tris-HCL at pH 7.5, $150 \mathrm{mM}$ $\mathrm{NaCl}, 5 \mathrm{mM} \mathrm{MgCl}, 2 \mathrm{mM}$ DTT), at $37^{\circ} \mathrm{C}$ for $1 \mathrm{~h}$. Samples were mixed with Laemmli sample buffer, heated at $95^{\circ} \mathrm{C}$ for $5 \mathrm{~min}$, and electrophoresed on SDS-PAGE. Following electrophoresis, proteins were transferred to cellulose nitrate membranes and detected by autoradiography. Purified p $73 \alpha$ was generated by immunoprecipitation of in vitro-translated $\left[{ }^{35}\right.$ S $]$ methionine Flag-p73 $\alpha$ with Flag beads (Sigma), followed by elution with Flag peptide (Sigma).

\section{Coimmunoprecipitation studies}

Coimmunoprecipitation experiments from cell extracts and from in vitro ${ }^{35}$ S-labeled proteins were carried out as previously described (Asher et al. 2003).

\section{Trypsin digestion}

Trypsin digestion of $\left[{ }^{35} \mathrm{~S}\right]$ methionine Flag-p73 was carried out in digestion buffer (100 mM Tris-HCL at pH 7.5, $150 \mathrm{mM} \mathrm{KCl}, 0.1 \%$ NP40, $10 \%$ glycerol, $0.2 \mathrm{ng} / \mathrm{\mu L}$ trypsin [Sigma]), at $37^{\circ} \mathrm{C}$ for $10 \mathrm{~min}$. Samples were then mixed with Laemmli sample buffer, heated at $95^{\circ} \mathrm{C}$ for $5 \mathrm{~min}$, electrophoresed on SDS-PAGE, and detected by autoradiography.

\section{Proteasome purification and analysis}

Mouse livers were homogenized in buffer containing $20 \mathrm{mM}$ Tris-HCL (pH 7.5), $1 \mathrm{mM}$ EDTA, $1 \mathrm{mM}$ DTT, and $250 \mathrm{mM}$ sucrose. Following $38 \%-70 \%$ ammonium sulfate precipitation and centrifugations, the precipitate was dissolved in buffer containing $20 \mathrm{mM}$ Tris-HCL (pH 7.5), 1 mM DTT, $20 \%$ glycerol, and loaded on a Sepharose $6 \mathrm{~B}$ column. Fractions of $2 \mathrm{~mL}$ were collected and analyzed for the presence of NQO1 and 20S proteasomes by Western blot analysis. The proteasome-containing fractions were combined and loaded onto a Resource-Q column. Elution was performed with $0.35-0.4 \mathrm{M} \mathrm{NaCl}$, and samples were concentrated and loaded on a $10 \%-40 \%$ glycerol gradient. The purified proteasomes were analyzed by SDS-polyacrylamide gels or by nondenaturing polyacrylamide gel (Glickman et al. 1998). Proteasome peptidase activity was determined by their ability to hydrolyze the flurogenic peptide sucLLVY-AMC as previously described (Glickman et al. 1998).

\section{Purification of recombinant NQO1}

pET28-His-TEV-NQO1 was expressed in bacteria, and the bacteria were then lysed by sonication in $50 \mathrm{mM}$ Tris-HCL (pH 7.5), $150 \mathrm{mM} \mathrm{NaCl}$, and $1 \mathrm{mM}$ PMSF. Soluble His-TEV-NQO1 was purified using a Ni-NTA column (HiTrap chelating HP) followed by gel-filtration chromatography (HiLoad 16/60 superdex 200). Purified His-TEV-NQO1 was further cleaved by TEV protease, and the His-TEV was removed upon binding to a Ni-NTA column.

\section{Acknowledgments}

We thank Dr. Y. Reiss for the purified 20S, S. Budilovsky and V. Reiss for their assistance, and Dr. J. Lotem and A. Cooper for their critical review and advice. This work was supported by a research grant from the Israel Academy of Sciences and Humanities to C.K. and to Y.S., and a grant to Y.S. from the Laub Fund for Oncogene Research.

\section{References}

Anwar, A., Dehn, D., Siegel, D., Kepa, J.K., Tang, L.J., Pietenpol, J.A., and Ross, D. 2003. Interaction of human NAD(P)H:quinone oxidoreductase 1 (NQO1) with the tumor suppressor protein p53 in cells and cell-free systems. I. Biol. Chem. 278: 10368-10373. 
Asher, G., Lotem, J., Cohen, B., Sachs, L., and Shaul, Y. 2001. Regulation of p53 stability and p53-dependent apoptosis by NADH quinone oxidoreductase 1. Proc. Natl. Acad. Sci. 98: 1188-1193.

Asher, G., Lotem, J., Kama, R., Sachs, L., and Shaul, Y. 2002a. NQO1 stabilizes p53 through a distinct pathway. Proc. Natl. Acad. Sci. 99: 3099-3104.

Asher, G., Lotem, J., Sachs, L., Kahana, C., and Shaul, Y. 2002b. Mdm-2 and ubiquitin-independent $\mathrm{p} 53$ proteasomal degradation regulated by NQO1. Proc. Natl. Acad. Sci. 99: 13125-13130.

Asher, G., Lotem, J., Tsvetkov, P., Reiss, V., Sachs, L., and Shaul, Y. 2003. P53 hot-spot mutants are resistant to ubiquitin-independent degradation by increased binding to $\mathrm{NAD}(\mathrm{P}) \mathrm{H}$ :quinone oxidoreductase 1 . Proc. Nat1. Acad. Sci. 100: 15065-15070.

Asher, G., Lotem, J., Sachs, L., and Shaul, Y. 2004. p53-dependent apoptosis and NAD(P)H:quinone oxidoreductase 1. Methods Enzymol. 382: 278-293.

Chowdary, D.R., Dermody, J.J., Jha, K.K., and Ozer, H.L. 1994. Accumulation of p53 in a mutant cell line defective in the ubiquitin pathway. Mol. Cell. Biol. 14: 1997-2003.

Coux, O., Tanaka, K., and Goldberg, A.L. 1996. Structure and functions of the 20S and 26S proteasomes. Annu. Rev. Biochem. 65: 801-847.

Friguet, B., Bulteau, A.L., Conconi, M., and Petropoulos, I. 2002. Redox control of 20S proteasome. Methods Enzymol. 353: 253-262.

Glickman, M.H., Rubin, D.M., Coux, O., Wefes, I., Pfeifer, G., Cjeka, Z., Baumeister, W., Fried, V.A., and Finley, D. 1998. A subcomplex of the proteasome regulatory particle required for ubiquitin-conjugate degradation and related to the COP9-signalosome and eIF3. Cell 94: 615623.

Goldberg, A.L. 2003. Protein degradation and protection against misfolded or damaged proteins. Nature 426: 895-899.

Haupt, Y., Maya, R., Kazaz, A., and Oren, M. 1997. Mdm2 promotes the rapid degradation of p53. Nature 387: 296-299.

Hershko, A. 1996. Lessons from the discovery of the ubiquitin system. Trends Biochem. Sci. 21: 445-449.

Hershko, A. and Ciechanover, A. 1998. The ubiquitin system. Annu. Rev. Biochem. 67: 425-479.

Hosoda, S., Nakamura, W., and Hayashi, K. 1974. Properties and reaction mechanism of DT diaphorase from rat liver. J. Biol. Chem. 249: 64166423.

Kubbutat, M.H., Jones, S.N., and Vousden, K.H. 1997. Regulation of p53 stability by Mdm2. Nature 387: 299-303.

Long II, D.J., Gaikwad, A., Multani, A., Pathak, S., Montgomery, C.A., Gonzalez, F.J., and Jaiswal, A.K. 2002. Disruption of the NAD(P)H:quinone oxidoreductase 1 (NQO1) gene in mice causes myelogenous hyperplasia. Cancer Res. 62: 3030-3036.

Ma, Q., Cui, K., Xiao, F., Lu, A.Y., and Yang, C.S. 1992. Identification of a glycine-rich sequence as an $\mathrm{NAD}(\mathrm{P}) \mathrm{H}$-binding site and tyrosine 128 as a dicumarol-binding site in rat liver $\mathrm{NAD}(\mathrm{P}) \mathrm{H}$ :quinone oxidoreductase by site-directed mutagenesis. J. Biol. Chem. 267: 22298-22304.

Mamroud-Kidron, E., Omer-Itsicovich, M., Bercovich, Z., Tobias, K.E., Rom, E., and Kahana, C. 1994. A unified pathway for the degradation of ornithine decarboxylase in reticulocyte lysate requires interaction with the polyamine-induced protein, ornithine decarboxylase antizyme. Eur. J. Biochem. 226: 547-554.

Pickart, C.M. and Cohen, R.E. 2004. Proteasomes and their kin: Proteases in the machine age. Nat. Rev. Mol. Cell. Biol. 5: 177-187.

Verma, R. and Deshaies, R.J. 2000. A proteasome howdunit: The case of the missing signal. Cell 101: 341-344.

Vogelstein, B., Lane, D., and Levine, A.J. 2000. Surfing the p53 network. Nature 408: 307-310.

Zhang, F., Su, K., Yang, X., Bowe, D.B., Paterson, A.J., and Kudlow, J.E. 2003. O-GlcNAc modification is an endogenous inhibitor of the proteasome. Cell 115: 715-725. 


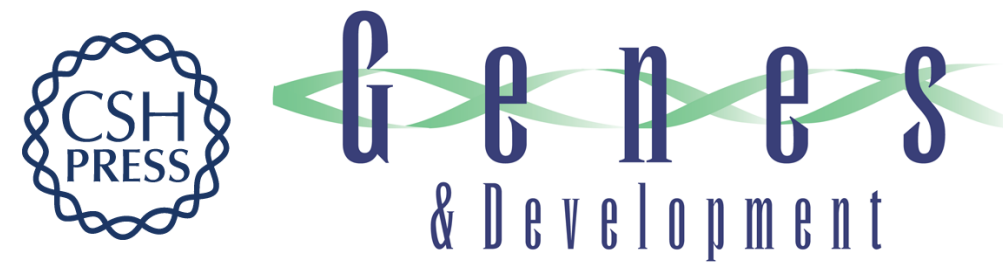

\section{A mechanism of ubiquitin-independent proteasomal degradation of the tumor suppressors p53 and p73}

Gad Asher, Peter Tsvetkov, Chaim Kahana, et al.

Genes Dev. 2005, 19:

Access the most recent version at doi:10.1101/gad.319905

Supplemental
Material http://genesdev.cshlp.org/content/suppl/2005/01/13/19.3.316.DC1

References This article cites 23 articles, 9 of which can be accessed free at: http://genesdev.cshlp.org/content/19/3/316.full.html\#ref-list-1

License

Email Alerting Receive free email alerts when new articles cite this article - sign up in the box at the top Service right corner of the article or click here.

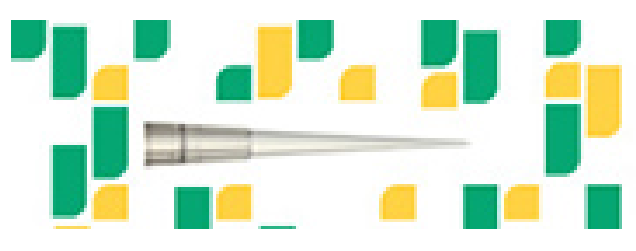

Focused on your science. 